\title{
Mortality among free-ranging California condors (Gymnogyps californianus) during 2010-2014 with determination of last meal and toxicant exposure
}

\author{
Tabitha Viner1,4 DVM DACVP, Rebecca Kagan' DVM DACVP, Bruce Rideout² DVM PhD DACVP, Ilse Stalis² DVM, Rebecca \\ Papendick² DVM DACVP, Allan Pessier ${ }^{3}$ DVM DACVP, Margaret E Smith ${ }^{1}$ MS, Mary Burnham-Curtis ${ }^{1}$ PhD, Brian Hamlin ${ }^{1}$ BS
}

\begin{abstract}
Over the past 30 years, the California condor (Gymnogyps californianus) population has rebounded from 22 individuals to more than 200 birds living in the wild. Historical impacts on the population have been mostly anthropogenic. In this study, we explore mortality and cause of death data from condors that died during the years 2010-2014 and compare these to mortality data described by Rideout et al. in 2012, covering the years 1992-2009. Also, morphologic and genetic analysis of the upper gastrointestinal (GI) tract contents was performed on the 2010-2014 condor mortalities to determine animal origins of the last meal eaten. The maximum population at risk within this time frame was 329 birds. During this time, 88 condors died and underwent postmortem examination, and 41 birds were lost to tracking efforts and presumed dead (39\% crude mortality rate; 129/329). A cause of death was determined for 66 of the 88 necropsied birds. Lead toxicosis remained a significant negative factor in condor population recovery, being related to the deaths of 37 adult and juvenile condors (56\% proportional mortality rate). Cattle were less often part of the last meal of lead-intoxicated condors than condors that succumbed to other causes of death. Based on these data, continued efforts to mitigate the impact of lead on California condors should be pursued.
\end{abstract}

KEYWORDS: veterinary forensic sciences, lead, diet, pathology, genetics, toxicology, California condor, Gymnogyps californianus

California condors (CACO; Gymnogyps californianus) are a critically endangered species currently indigenous to the southwestern United States and Baja peninsula of Mexico. Once wide-ranging, the condor population steadily declined during the 20th century due to hunting, habitat encroachment, the effects of DDT exposure, and lead intoxication (Burnett et al. 2013; Church et al. 2006; Rideout et al. 2012). Conservation efforts undertaken in the 1960s and 1970s included radio-tracking of wild condors and captive rearing of wild-laid eggs (Wilbur 1978). Despite these efforts, the wild population continued to decline. To save the species from extinction, the 22 condors that remained in the wild in 1987 were collected for inclusion in a captive breeding program. As of 2017, the total population had risen to 463 individuals, of which 290 were wild, free-ranging birds (U.S. Department of the Interior
2017). Contamination of the environment by spent lead ammunition continues to negatively impact the full and independent recovery of the California condor (Church et al. 2006; Kelly et al. 2014).

The purpose of this study was to explore the postmortem findings of free-ranging CACO that either died in the wild or under temporary holding from 2010 to 2014. Mortality of this population was compared to that described by Rideout et al. (2012), which was composed of the free-ranging CACO population that died between 1992 and 2009, inclusive. Health monitoring, supplemental feeding, and breeding management of CACO were ongoing during both studies. Also, animals comprising the last meal of the 2010-2014 condors were determined and applied to liver and bone lead evaluation results.

\footnotetext{
1 U.S. Fish and Wildlife Service National Forensics Laboratory, 1490 East Main Street, Ashland, OR 97520-1310 USA

2 Disease Investigations, Institute for Conservation Research, San Diego Zoo Global, PO Box 120551, San Diego, CA $92112-0551$ USA

3 Washington Animal Disease Diagnostic Laboratory, Washington State University, PO Box 647034, Pullman, WA 99164-7034 USA

Corresponding author: Tabitha Viner, tabitha_viner@fws.gov, 541-488-6505
} 


\section{Materials and Methods}

All CACO are tagged and observed from egg-laying to death (Finkelstein et al. 2012). At the appropriate age, each bird is fitted with a colored wing tag on which a unique identifying number is printed. CACO also are implanted with a transponder and fitted with a transmitter that emits a mortality signal when the bird has not moved during a set time interval. Thus, the age of the bird at death can be definitively determined or reliably estimated.

All condors that were found dead in the wild or died under temporary holding during the study period underwent postmortem examination by board-certified veterinary pathologists at either the San Diego Zoo or the National Fish and Wildlife Forensics Laboratory (NFWFL). Historical data, including antemortem blood lead concentrations, demographic data, and clinical history, if available, were provided to the pathologists. If autolysis did not preclude such tests, the postmortem examinations included radiographs, a detailed evaluation of the tissues, appropriate ancillary diagnostic tests, and histologic examination. When adequate postmortem preservation allowed, the liver was tested for lead concentration. A tibiotarsal sample from most birds, starting in 2013, was tested for lead concentration. Determination of recent meal composition was made via genetic or morphological evaluation of crop or ventriculus contents.

If metal was present in the GI tract and large enough for retrieval, the suspected firearm projectile components were examined using microscopic and macroscopic techniques to determine the size or caliber of the bullet or shot pellet material. The metal composition of the particles was determined using an Orbis PC Micro X-ray Fluorescence Analyzer (EDAX, Mahwah, NJ). In cases where metal particles were too small for retrieval, the unique radiographic morphology of lead firearm projectile fragments was used for diagnosis. The irregular shape and varying size of bright, metal densities are characteristic of bullet fragments, while spherical or semi-spherical bright, metal densities indicate shot pellets.

Franson (1996) examined non-waterfowl birds and described liver lead concentrations below 6 parts per million (ppm) wet weight (WW) as subclinical lead exposure and toxicosis at concentrations above this level. Factors considered in the current study when evaluating CACO for lead toxicosis included a recent diagnosis of and treatment for lead toxicosis, recent antemortem blood lead concentration above $0.5 \mathrm{ppm}$, lead fragments within the GI tract, muscle and fat atrophy, and postmortem liver lead concentration above $6 \mathrm{ppm}$ WW. Elevated concentrations of lead in bone were considered to be suggestive of lead toxicosis, but not a sole determining factor.
Bone for lead analysis was prepared by microwave acid digestion using the MARS microwave digester (CEM Corporation), and lead concentration was determined by flame atomic absorption spectrophotometry using a Thermo M6 with Solaar Analysis software and deuterium arc background. Standard operating procedures were suggested by AOAC International and Thermal Jarrell Ash Corporation. Lead concentrations (ppm) were relayed on a dry weight (DW) basis. Lead quantitation in livers (Behmke et al. 2015) was determined on an Agilent 7500ce ICP-MS metal analyzer (Agilent Technologies, Santa Clara, CA) equipped with a Cetac autosampler (Cetac, Omaha, NE) and MicroMist nebulizer (Agilent). The instrument was calibrated with appropriate dilutions of Specpure 1000- $\mu \mathrm{g} /$ $\mathrm{mL}$ stock solutions (Alfa Aesar, Ward Hill, MA) of each element of interest according to in-house generated standard operating procedures derived from the operator's manual (Agilent Technologies 2004). Each quantitative analysis used the National Institute of Standards and Technology standard reference material (NIST SRM) for matrix-matched quality control. Accordingly, analyses performed on tissue were matched to NIST SRM 1577c (bovine liver). Digested samples were diluted in an aqueous solution of $0.05 \%(\mathrm{w} / \mathrm{v})$ ethylenediaminetetraacetic acid (EDTA), 1\% (w/v) ammonium hydroxide, 0.05\% (w/v) Triton X-100 detergent, and $2 \%(\mathrm{w} / \mathrm{v})$ butanol prior to analysis. Listed reagents were from Sigma-Aldrich (St. Louis, MO). Limits of quantitation for lead were 1 part per billion (ppb).

DNA extraction of crop or ventriculus contents was completed with the Qiagen DNeasy kit on the Qiagen QIAxtractor robotic platform. Negative controls were included in each extraction run. A universal primer set (H15149-L14724) was used to amplify a mitochondrial DNA segment of approximately 450 base pairs that codes for cytochrome $b$ (Kocher et al. 1989). The heavy strand primer was labeled with an M13 tag to allow sequencing with a universal M13 sequencing primer in one direction. PCR was performed in a total volume of $50 \mu \mathrm{L}$, which contained $0.1 \mu \mathrm{L}$ Titanium Taq polymerase (Clonetech, Inc.), $1 \times$ PCR buffer and $3 \mathrm{mM} \mathrm{MgCl} 2$ (supplied with polymerase), $200 \mu \mathrm{M}$ dNTPs, $1 \mu \mathrm{M}$ of each forward and reverse primer, and $5 \mu \mathrm{L}$ of genomic DNA $(0.1-15 \mathrm{ng} / \mu \mathrm{L})$. PCR cycling parameters were as follows: initial denaturation at $95^{\circ} \mathrm{C}$ for $2 \mathrm{~min}, 30$ cycles of denaturation at $95^{\circ} \mathrm{C}$ for $1 \mathrm{~min}$, annealing at $52^{\circ} \mathrm{C}$ for $45 \mathrm{~s}$, elongation at 72 ${ }^{\circ} \mathrm{C}$ for $45 \mathrm{~s}$, final elongation at $72{ }^{\circ} \mathrm{C}$ for $5 \mathrm{~min}$. DNA sequencing was performed on purified PCR amplicons by the dideoxynucleotide sequencing method (Sanger et al. 1977) with the Life Technologies BigDye Terminator v3.1 cycle sequencing kit. Resulting sequences were analyzed by capillary electrophoresis on an Applied Biosystems 3500xL system. Positive and negative controls were run with all PCRs, including amplification and sequencing a segment of the pGem vector as a positive sequencing control. 
Sequences were imported into the Geneious R6 software package (Biomatters, LLC) for editing and primer endtrimming before comparative alignment to sequences in the NFWFL mammal sequence database, which is comprised of publicly available sequences (e.g., GenBank), sequences from in-house vouchered reference materials, and sequences from adjudicated forensic casework samples. Species' origin of unknown samples was determined by a direct comparison of the unknown sample's nucleotide sequence with the database sequences. Published phylogenies used to validate forensic protocols establish the criteria by which unknown samples are assigned to species. Samples that do not meet the criteria are assigned to the next highest taxonomic level (e.g., genus or family). Species assignments were made with $99 \%$ or higher sequence similarity with the reference sequence.

Standard morphological protocols were used to determine the taxonomic identification of hair retrieved from crop and ventriculus contents (Moore et al. 1974; Tridico et al. 2014). The analysis consisted of microscopically extracting those anatomical elements that offered diagnostic details at the lowest taxonomic level possible. These anatomical elements were compared to reference materials in the NFWFL collection.

\section{Results and Discussion}

Of 329 CACO living in the wild during all or part of the study period (2010-2014), 88 died and underwent a necropsy, and 41 went missing and are presumed to be dead (39\% crude mortality rate). In this cohort of 129 birds, 53 were adults ( $\geq 6$ years of age), 54 were juveniles ( 6 months to $<6$ years of age), and 22 were nestlings ( $<6$ months old). A comparison to the cohort described by Rideout et al. in 2012 is shown in TABLE 1. As seen in this study, as the CACO population recovers and ages, a proportional increase in mortality among adults is expected.

TABLE 1-Mortality of the free-ranging California condor (Gymnogyps californianus) population during 2010-2014 compared to that in 1992-2009. The percentage of adult mortalities in the current data set likely reflects the aging population of this managed species.

\begin{tabular}{lccccc}
\hline & \multicolumn{2}{c}{$\begin{array}{c}2010-2014 \\
(n=129)\end{array}$} & & & \multicolumn{2}{c}{$\begin{array}{c}1992-2009 \\
(n=135)\end{array}$} \\
\cline { 2 - 3 } \cline { 6 - 7 } Lifestage & $\%$ & $n$ & & $\%$ & $n$ \\
\hline adult & 41 & 53 & & 17 & 23 \\
juvenile & 42 & 54 & & 71 & 96 \\
nestling & 17 & 22 & & 12 & 16 \\
\hline
\end{tabular}

A cause of death could be determined for 66 of the 88 (75\%) birds necropsied. Postmortem decomposition and scavenging were confounding factors in the determination of mortality data. Proportional mortality rates (PMRs) for individual data sets were calculated by dividing the number of mortalities due to a specific cause by the overall number of mortalities of a known cause (Rideout et al. 2012). For this study set, causes of death were known, and PMRs were determined for 34 adults, 24 juveniles, and 8 nestlings.

In the current study, lead toxicosis was considered to play a role in the deaths of 37 condors, including 29 adults (85\% PMR, 29/34) and 8 juveniles (33\% PMR, 8/24; 64\% combined PMR). Rideout et al. (2012) described 23 adults and juveniles with lead toxicosis and a combined PMR among these age groups of 35\% during the 1992-2009 period. Of the 37 condors with lead toxicosis in the current study, 13 died while under treatment for elevated lead concentrations discovered during routine field monitoring practices (Sorenson and Burnett 2007). The remaining 24 birds were either found dead $(n=22)$ or died during capture and transport $(n=2)$.

Predation was the cause of death of 3 of the 5 adults that did not die of lead toxicosis. Two adults died of blunt force trauma of an undetermined etiology. Lead concentrations in these 5 birds were less than 1 ppm WW.

Of the 37 lead-intoxication mortalities, 19 had metal densities in the GI tract, evident in postmortem radiographs. Fourteen $(61 \%)$ of the 23 birds that had died in the field had radiographically evident metal in the crop or ventriculus. Most metal densities were consistent with small ballistic particulates resulting from the fragmentation of lead bullets (FIGURE 1), but 3 birds had ingested lead shot pellets, and 2 had largely intact bullets. The presence of lead fragments in the GI tract at the time of death may indicate an acute, marked response to lead ingestion by CACO, or it may manifest the effect of lead on the motility of the upper GI tract, resulting in prolonged retention of lead fragments. Alternatively, condors that die after recent ingestion of lead may already have had high circulating concentrations of the toxin, predisposing them to acute mortality through the addition of another lead-contaminated meal.

Condors that died with lead toxicosis had liver lead concentrations that ranged from 4.6-130 ppm WW, with a mean of $31.6 \mathrm{ppm} \mathrm{WW} \mathrm{(SD,} \mathrm{25.7).} \mathrm{One} \mathrm{condor} \mathrm{that} \mathrm{died}$ due to contact with a live power line had a liver lead concentration of $52.49 \mathrm{ppm}$ WW. In this case, lead toxicosis was determined to be the proximate cause of death, and electrocution, the immediate cause of death. Another condor died after 2 weeks of lead chelation therapy with a liver lead concentration of $4.6 \mathrm{ppm}$ WW. Lead toxicosis was diagnosed as the proximate cause of death in this case because this condition began the chain of events that lead to the bird's death. Birds with other mortality factors had liver lead concentrations from 0.03-1.9 ppm WW, a mean of $0.23 \mathrm{ppm}$ WW (SD, 0.47). 


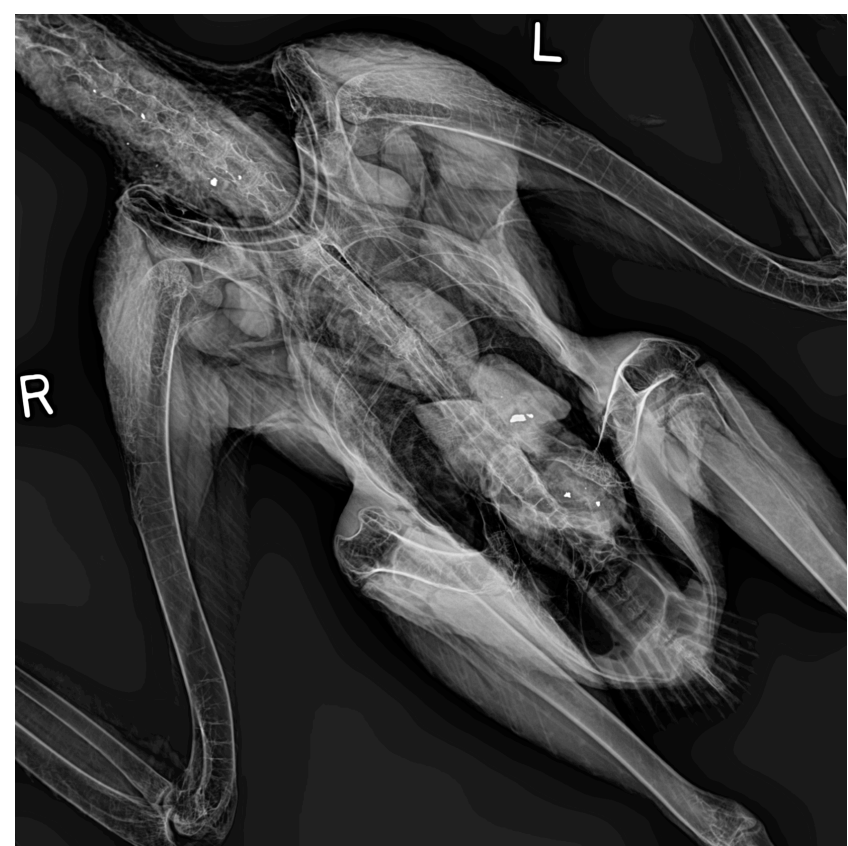

FIGURE 1-Irregular, lead fragments are present in the crop and ventriculus of this California condor (Gymnogyps californianus). Liver lead concentration was 42.22 ppm wet weight. Muscle and hair within the crop were both determined to be of deer origin.

Components of the last meal were determined for 20 condors that died of lead toxicosis in the wild and 21 birds that died of other causes (TABLE 2). The variability of gut content character required the use of genetic and morphological techniques to identify the last meal. The most common food item taxon in this group was cervid (6), followed by equid (4), caprid (3), canid (2), bovid (1), and suid (1). One bird that had eaten deer had also fed on a goat before death. Cattle were more heavily represented in field mortalities not associated with lead toxicosis. Of the 21 birds that died of non-lead causes for which last meal was determined, bovid tissue was present in 10; cervid in 5; suid in 3; equid in 2; and leporid, canid, and ursid each in 1 . Two different animals were present in the GI contents of 7 of these birds, and 1 bird had fed on 3 different species before death. These findings are similar to those of Kelly et al. (2014), which showed that increased independence from proffered feeding (generally bovid) was associated with a higher incidence of elevated blood lead concentrations during routine testing.

Ingestion of lead ammunition is a well-established cause of lead toxicosis and mortality in CACO (Rideout et al. 2012; Kelly et al. 2014). Primary vehicles of transmission have been assumed to be hunter-killed deer or pigs within the CACO range. Data presented here introduce the possibility that gunshot-killed horses and small domestic livestock may remain in the field and thus are available to opportunistic scavengers, such as condors.
TABLE 2-Last meal components for California condors (Gymnogyps californianus) that died due to lead toxicosis compared to other causes of death. Data are related to birds that died in the wild without benefit of treatment or chelation. Some birds had eaten more than one type of animal.

\begin{tabular}{lcc}
\hline & \multicolumn{2}{c}{ Cause of death } \\
\cline { 2 - 3 } Animal & $\begin{array}{c}\text { Lead toxicosis } \\
(n=20)\end{array}$ & $\begin{array}{c}\text { Other } \\
(n=21)\end{array}$ \\
\hline cervid & 6 & 5 \\
equid & 4 & 2 \\
canid & 2 & 1 \\
caprid & 3 & 0 \\
bovid & 1 & 10 \\
suid & 1 & 3 \\
ursid & 0 & 1 \\
leporid & 0 & 1 \\
unspecified mammal & 4 & 5 \\
\hline
\end{tabular}

Bone lead concentrations were determined for 33 birds in this study set and ranged from $<0.25$ to $71.17 \mathrm{ppm} \mathrm{DW}$, with a mean of $18.9 \mathrm{ppm}$ DW (SD, 17.5). Concentrations of lead in bone reflect exposure to the heavy metal for months to years and may increase with additional exposure. Lead also accumulates in the bones of some avian species during times of high calcium demand, such as preparation for egglaying (Finley and Dieter 1978) or early growth (Strom et al. 2005). In the lead toxicity group, females $(F, n=19)$ and males $(M, n=18)$ were almost equally represented, and mean bone lead levels in each sex were similar $(F=31.75$ ppm WW, $M=33.24$ ppm WW, $P=0.89$ ). Additionally, sex was not a significant distinction between birds that died of lead toxicity and those that died of other causes $\left(\chi^{2}\right.$ test; $P=$ 0.31 ), which suggests that the metabolic processes involved in reproduction are not a predisposing factor for lead toxicity. Bone lead concentrations are considered to be relatively static, with minor reductions occurring only during bone remodeling. Weak positive correlation occurred between lead concentration in the bone and time between first release into the wild and mortality (FIGURE 2; $r=0.30$ ). Twenty-one of these birds with measured bone lead concentrations also had recorded liver lead concentrations. Positive correlation occurred between postmortem bone and liver lead concentrations (FIGURE 3; $r$ $=0.53$ ).

Trash ingestion remained an issue in the nestling subpopulation of this study. Of 14 nestlings examined postmortem, 8 had pieces of glass, plastic, or rubber in the GI tract. The foreign material did not appear grossly or microscopically to damage the lining of the crop, esophagus, proventriculus, or ventriculus, nor was it directly and definitively linked to the mortality of the birds. A cause of death could not be determined for 6 nestlings. Trauma, either parental or predatory (golden eagles), 
caused the deaths of 6 nestlings (75\% PMR, 6/8). One nestling died of septicemia, and one succumbed to iatrogenic causes. The relationship between trash ingestion, behavioral abnormalities, and parental treatment or attention could not be assessed. Behavioral abnormalities resulting from the ingestion of foreign objects may predispose nestlings to trauma from parents or predators.

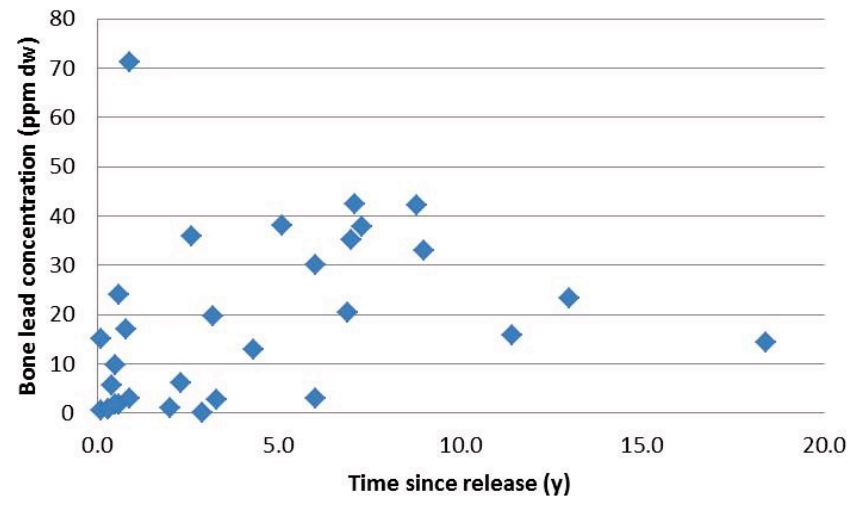

FIGURE 2-Positive association $(r=0.30)$ between lead concentration in the bones of wild California condors (Gymnogyps californianus) and the time between the first release into the wild and the death of the bird. ppm dw, parts per million on a dry weight basis; $y$, years.

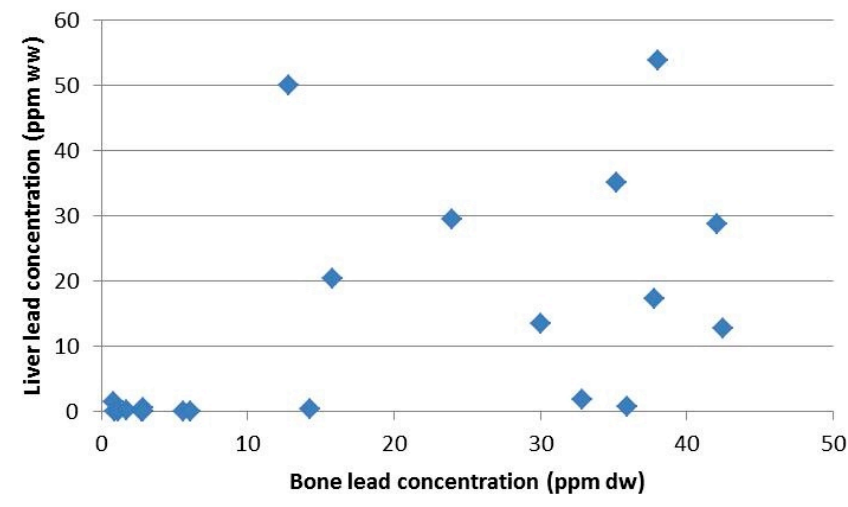

FIGURE 3-The concentration of stored lead in the bone compared to that in the liver of these California condors (Gymnogyps californianus). An overall positive correlation ( $r=$ 0.53 ) is shown between bone and liver lead concentrations. ppm, parts per million; ww, on a wet weight basis; dw, on a dry weight basis.

Trauma was the most significant cause of death in the juvenile age group (39\% PMR, 11/28). Eight of these traumatic deaths were due to predation. In one condor, genetic analysis of fatal bite marks on the neck revealed bobcat DNA. Juveniles that died of traumatic injuries had measured liver lead concentrations between $<0.5-1.44 \mathrm{ppm}$ WW. Other causes for juvenile deaths included gunshot (2), drowning (1), and upper respiratory tract infection (1).

Based on these data, lead toxicosis remains a significant, negative influence on the recovery of the CACO population, and was the leading cause of death of adults in our study group. Condors scavenge various wild and domestic species, any of which may harbor toxicants such as lead. Continued monitoring of CACO is warranted, and efforts to mitigate access to and the effects of lead should be continued.

\section{Acknowledgements}

The authors wish to sincerely thank the many pathologists, veterinarians, biologists, and technicians involved in the many aspects of the condor recovery program for their efforts to elevate the health of the California condor population. The authors gratefully acknowledge $J$ Buckner and $J$ Lankton of the USGS National Wildlife Health Center, and J Buchweitz of the Michigan State University Veterinary Diagnostic Laboratory for lead testing services; D J Straughan, J P LeMay, and B C Yates for genetic and morphological analysis; and P J McClure, and A D Reinholz for chemical and metal analysis.

\section{Disclosures}

The findings and conclusions in this article are those of the authors and do not necessarily represent the views of the U.S. Fish and Wildlife Service. The mention of tradenames does not imply U.S. government endorsement of commercial products.

\section{References}

Behmke S, Fallon J, Duerr AE, Lehner A, Buchweitz J, Katzner T. 2015. Chronic lead exposure is epidemic in obligate scavenger populations in eastern North America. Environ Int. 79:51-5. doi:10.1016/ j.envint.2015.03.010.

Burnett LJ, Sorenson KJ, Brandt J, Sandhaus EA, Ciani D, Clark M, David C, Theule J, Kasielke S, Risebrough RW. 2013. Eggshell thinning and depressed hatching success of California condors reintroduced to central California. The Condor. 115:477-91. doi:10.1525/ cond.2013.110150.

Church MR, Gwiazda R, Risebrough RW, Sorenson K, Chamberlain CP, Farry S, Heinrich W, Rideout BA, Smith DR. 2006. Ammunition is the principal source of lead accumulated by California condors reintroduced to the wild. Environ Sci Technol. 40(19):6143-50. doi:10.1021/es060765s.

Finkelstein ME, Doak DF, George D, Burnett J, Brandt J, Church M, Grantham J, Smith DR. 2012. Lead poisoning and the deceptive recovery of the critically endangered California condor. Proc Natl Acad Sci U S A. 109(28):11449-54. doi:10.1073/pnas.1203141109.

Finley MT, Dieter MP. 1978. Influence of laying on lead accumulation in bone of mallard ducks. J Toxicol Environ Health. 4:123-9. doi:10.1080/15287397809529649.

Franson JC. 1996. Interpretation of tissue lead residues in birds other than waterfowl. In: Beyer WN, Heinz GH, Redmon-Norwood AW, editors. Environmental contaminants in wildife: interpreting tissue concentrations. Boca Raton (FL): CRC Press. p. 265-79.

Kelly TR, Grantham J, George D, Welch A, Brandt J, Burnett LJ, Sorenson KJ, Johnson M, Poppenga R, Moen D, Rasico J, Rivers JW, Battistone C, Johnson CK. 2014. Spatiotemporal patterns and risk 
factors for lead exposure in endangered California condors during 15 years of reintroduction. Conserv Biol. 28(6):1721-30. doi:10.1111/ cobi.12342.

Kocher TD, Thomas WK, Meyer A, Edwards SV, Paabo S, Villablanca FX, Wilson AC. 1989. Dynamics of mitochondrial DNA evolution in animals: amplification and sequencing with conserved primers. Proc Natl Acad Sci U S A. 86(16):6196-200. doi:10.1073/pnas.86.16.6196.

Moore TD, Spence LE, Dugnolle CE. 1974. Identification of the dorsal guard hairs of some mammals of Wyoming. Laramie (WY): Wyoming Game and Fish Department.

Rideout BA, Stalis I, Papendick R, Pessier A, Puschner B, Finkelstein ME, Smith DR, Johnson M, Mace M, Stroud R, Brandt J, Burnett J, Parish C, Petterson J, Witte C, Stringfield C, Orr K, Zuba J, Wallace M, Grantham J. 2012. Patterns of mortality in free-ranging California condors (Gymnogyps californianus). J Wildlife Dis. 48(1):95-112. doi:10.7589/0090-3558-48.1.95.

Sanger F, Nicklen S, Coulson AR. 1977. DNA sequencing with chainterminating inhibitors. Proc Natl Acad Sci U S A. 74(12):5463-7. doi:10.1073/pnas.74.12.5463.
Sorenson KJ, Burnett LJ. 2007. Lead concentrations in the blood of Big Sur California condors. In: Mee A, Hall LS, editors. California condors in the 21st century. Washington (DC): American Ornithologists' Union and Nuttall Ornithological Club. (Series in ornithology; vol. 2). p 18595.

Strom SM, Patnode KA, Langenberg JA, Bodenstein BL, Scheuhammer AM. 2005. Lead contamination in American woodcock (Scolopax minor) from Wisconsin. Arch Environ Contam Toxicol. 49(3):396-402. doi:10.1007/s00244-005-7063-0.

Tridico SR, Houck MM, Kirkbride KP, Smith ME, Yates BC. 2014. Morphological identification of animal hairs: myths and misconceptions, possibilities and pitfalls. Forensic Sci Int. 238:101-7. doi:10.1016/j.forsciint.2014.02.023.

U.S. Department of the Interior. 2017. California Condor recovery program: 2017 annual population status. Washington (DC): U.S. Fish and Wildlife Service [accessed 2019 Sep]. fws.gov/cno/es/CalCondor/ PDF_files/2017-CA-condor-population-status.pdf.

Wilbur SR. 1978. The California condor, 1966-76: a look at its past and future. North Am Fauna. 72:1-136. 